\title{
Vitamin D Deficiency in Association with HSCRP Linked to Obesity
}

\author{
Dr. Pushpamala Ramaiah ${ }^{1}$, Dr. Ayman Johargy², \\ Dr. Lamiaa Ahmed Elsayed ${ }^{3}$, Dr. Grace Lindsey ${ }^{4}$ \\ ${ }^{1,4}$ Professor, ${ }^{2}$ Associate Professor of Medical Microbiology, Faculty of Medicine, \\ ${ }^{3}$ Associate Professor at Faculty of Nursing \\ 1,2,3,4 Umm al Qura University, Makkah, Kingdom of Saudi Arabia
}

\begin{abstract}
Vitamin D deficiency is gaining increasing attention for its novel association with Obesity. Studies have found that maintaining vitamin $\mathrm{D}$ status may reduce ones risk of developing various diseases. Background In 2010, overweight and obesity were estimated to cause 3.4 million deaths, $3 \cdot 9 \%$ of years of life lost, and $3 \cdot 8 \%$ of disability-adjusted life-years (DALYs) worldwide. The rise in obesity has led to widespread calls for regular monitoring of changes in overweight and obesity prevalence in all populations. The treatment of obesity and cardiovascular diseases is one of the most difficult and important challenges nowadays. This paper seeks to examine the consistently reported relationship between obesity and low vitamin D concentrations in association with HSCRP, with reference to the possible underlying mechanisms. The possibility that vitamin D may assist in preventing or treating obesity is also examined and recommendations for future research are made. We tested the hypothesis that suggests Adults with severe obesity have lower 25-hydroxyvitamin D levels will have higher hs-CRP levels.
\end{abstract}

Keyword: Vitamin D deficiency, High Sensitive $C$ Reactive Protein, Obesity.

\section{INTRODUCTION}

Vitamin D deficiency is the most common nutritional deficiency and the very likely, the most common medical condition in the world. There is a consistent association in the published literature between increasing BMI and lower serum 25-hydroxyvitamin D (25D) concentrations. A bi-directional genetic study, which limits confounding, has suggested that higher BMI leads to lower 25D, with the effects of lower $25 \mathrm{D}$ on BMI likely to be small ${ }^{[1]}$. Lower vitamin $\mathrm{D}$ in obese people is a consistent finding across age, ethnicity, and geography. This may not always reflect a clinical problem. Obese people need higher loading doses of vitamin $\mathrm{D}$ to achieve the same serum 25-hydroxyvitamin D as normal weight. [ ${ }^{2}$ ]. His work suggests that vitamin D gets diluted throughout the body, and that dilution shows up as a deficiency in overweight and obese people, who have more mass than normal-weight people. On the other hand, a number of studies have confirmed that $\mathrm{C}$ reactive protein (CRP), an inflammatory marker, is a strong predictor of CVD Thus, it is likely feasible to prevent CVD with the help of CRP-reducing treatments. $^{[} 3{ }^{3}$. Previous studies have shown that vitamin D deficiency is associated with both developing obesity and the risk of obesity related complications, but studies on the effects of vitamin D supplementation have been inconclusive. The association between reduced $25 \mathrm{D}$ concentrations and obesity is therefore well-established, although the mechanisms for the lower 25D concentrations are not fully described, and there is uncertainty as to what the health consequences of these lower concentrations might be. This paper attempts to summarize the current state of knowledge regarding the causes of these reduced $25 \mathrm{D}$ concentrations, as well as the possible effects of vitamin D supplementation on obesity. ${ }^{[4]}$

Background: It has been estimated that 1 billion people worldwide have Vitamin D deficiency or insufficiency. This is mostly attributable to people getting less sun exposure because of climate, lifestyle, and concerns about skin cancer. There is a consistent association in the published literature between increasing BMI and lower serum 25-hydroxyvitamin 
D (25D) concentrations. ${ }^{[5]}$. Some people get tested for vitamin $\mathrm{D}$ and find, despite taking vitamin $\mathrm{D}$ regularly, that they are still not getting enough vitamin $\mathrm{D}$. This means their body is having hard time absorbing their vitamin D supplements. The vitamin D council recommends testing every three to six months, if they are trying different regimens. The vitamin $\mathrm{D}$ council believes that trying to get enough vitamin $\mathrm{D}$ from diet is unlikely to get sufficient vitamin D which is needed. ${ }^{[6]}$.Recently, there has been increased interest in the relevance for vitamin $\mathrm{D}$ to human health accompanied by increase demand of vitamin D testing, which has led to the use of less precise assays, which recommend the use of gold standard LC-MS assay, and insist a large national community based study of the prevalence of low vitamin D using this method. ${ }^{[7]}$. A1-Daggrey (2014) pointed out that the recommended period for sun exposure to the sun is half-an-hour, three to four days a week. There is a clear need for adequately-powered, prospective interventions which include baseline measurement of $25 \mathrm{D}$ concentrations and CRP also involve adequate doses of supplemental vitamin D. Until such studies have been reported, the role of vitamin D supplementation in obesity prevention remains uncertain.

\section{The aim of the study was:}

$>$ To identify the prevalence of vitamin D deficiency and HSCRP among adults.

$>$ To examine relationships between dietary habits and 25-hydroxyvitamin D [25(OH)D] level.

$>$ Investigate the associations between the $25(\mathrm{OH}) \mathrm{D}$ concentrations and the biomarker of inflammation HSCRP.

$>$ To evaluate whether vitamin D insufficiency and increased serum high-sensitivity C-reactive protein (hs-CRP) linked to Obesity.

Patients and Methods: Using a longitudinal, crosssectional design, systolic and diastolic blood pressure, dietary information, serum $25(\mathrm{OH}) \mathrm{D}$, serum CRP, fasting glucose and insulin, 2-h glucose from oral glucose tolerance test, hemoglobin A1c, thyroid profile, liver profile were recorded for 124 volunteer subjects at an referral clinic. Data was collected during their visit at clinic between April 2017 and April 2018. Adults of ages between 20 to 65 years were selected as participants, written informed consent was obtained from the subjects from whom data were collected prospectively. The subjects who were on use of any medication known to effect vitamin D metabolism were excluded from the project. Those subjects having $25(\mathrm{OH})$ vitamins D level $>150 \mathrm{ng} / \mathrm{mL}$ were also excluded from the study. Institutional Review Board approved the projects.

Tools of data collection: Data was collected by using: 1. A structured pre-validated questionnaire developed by King Saud University, Biomarkers Research Department after obtaining the consent from the author of the questionnaire. Pre-validated structured questionnaire was divided into three sections as follows: a. Socio demographic data. b. Polar questions with regard to knowledge about Vitamin D and associated diseases. c. MCQ about sunshine exposure and duration. 2. Measurement of Serum analysis to find out the level of Vitamin D, HSCRP and other biomarker values. The recommended, best test for assessing vitamin D status is to measure $25(\mathrm{OH})$ D levels using a reliable assay in population at risk for vitamin D deficiency is Electro chemical luminescence assay. 3. Anthropometric Assessment: Physical examination was performed by the physician and the nurse. Height and weight was taken using appropriate international standard scale. Waist circumference were measured using non stretchable tape, BMI was measured.

Statistical Analysis: Both descriptive and inferential statistical analysis was carried out using SPSS. Inferential statistics will include Independent $\mathrm{T}$ test/Mann Whitney $U$ Test, Paired sample T test/Wilcoxon Signed Rank test, multiple regression analysis/ Logistic regression, repeated measures ANOVA, Chi-Square test of independence. Paired sample t-test (two-tailed) or the repeated measures ANOVA, whichever is appropriate. Equivalent nonparametric analysis was used in case of violations of normality assumption.

Results: The prevalence of vitamin D insufficiency and deficiency were determined for the obese and non-overweight study population. Prevalence of vitamin $\mathrm{D}$ deficiency was defined by three different thresholds of $25(\mathrm{OH}) \mathrm{D}$, severe, moderate deficiency, deficiency and optimal level of vitamin D. Diagnostic criteria of vitamin D status is as follows [ 8 ] (Povoroznyuk.V. 2013) 
International Journal of Trend in Scientific Research and Development (IJTSRD) ISSN: 2456-6470

\begin{tabular}{|c|c|c|c|c|c|}
\hline Vitamin D & Severe deficiency & Deficiency & Insufficiency & Optimal level & Risk of Toxicity \\
\hline $\begin{array}{c}25(\mathrm{OH}) \mathrm{D} 3 \\
\text { Concentration }\end{array}$ & $0-10$ & $10-20$ & $20-30$ & $30-80$ & $>100$ \\
\hline
\end{tabular}

A $\chi 2$ test was used to compare the prevalence rate of vitamin $\mathrm{D}$ insufficiency and deficiency in the obese group to the non-overweight group.

In the present study records of 124 ostensibly healthy subjects enrolled for executive health check-up at different laboratories in India were chosen as research subjects. Amongst these, $77 \%$ were male and $23 \%$ female. The mean $25(\mathrm{OH})$ vitamin $\mathrm{D}$ level in the population was found to be $17.37 \pm 7.12 \mathrm{ng} / \mathrm{mL}$ (median 14.59) whereas upon segregation, in males the mean level was $18.96 \pm 10.36 \mathrm{ng} / \mathrm{mL}$ (median 17.76) and $14.24 \pm 6.79 \mathrm{ng} / \mathrm{mL}$ (median 13.72) in females $(p<0.0001)$.

\begin{tabular}{|c|c|c|c|c|c|c|}
\hline Study subjects(124) & $\%$ & Mean & SD & Severe Deficiency & Optimal Level & Chi square \\
\cline { 1 - 6 } Male & 77 & 18.96 & 10.36 & $18(51 \%)$ & $5(55 \%)$ & \multirow{2}{*}{.048} \\
\cline { 1 - 5 } Female & 47 & 14.24 & 6.79 & $17(49 \%)$ & $4(45 \%)$ & Not significant \\
\hline Total & 124 & - & - & 35 & 9 & \\
\hline
\end{tabular}

This result is not significant at $p<.05$. The reviews of (Babu\& Calvo 2012) documents supports our research findings of widespread vitamin D deficiency in Indian populations in higher and lower socioeconomic strata, in all age groups, in both genders and people in various professions. The subjects with Vitamin D deficiency were administered the recommended dose of Vitamin D and found the significant outcome in their serum level with the medianvalue of 31.28 (27.88-34.68) which was evident statistically by Overall increase by $68 \%$ of $25(\mathrm{OH})$ vitamin D level with no observed significant changes in the increased level of vitamin D between male and female. Serum $25(\mathrm{OH})$ vitamin $\mathrm{D}$ decreased with increasing age in all subjects $(\mathrm{r}=-0.29 ; \mathrm{P}<0.001)$.
On further evaluating these 124 subjects on the basis of their $25(\mathrm{OH})$ vitamin D status, $53.69 \%$ were found to have $25(\mathrm{OH})$ vitamin $\mathrm{D}$ severe deficiency $(<12 \mathrm{ng} / \mathrm{mL})$ which included $61 \%$ male and $39 \%$ female. Similarly $25(\mathrm{OH})$ vitamin $\mathrm{D}$ moderate insufficiency $(<20 \mathrm{ng} / \mathrm{mL})$ was observed in about $39 \%$ individuals including $52.5 \%$ male and $47.5 \%$ female. Only $7.31 \%$ of the total population had optimal $25(\mathrm{OH})$ vitamin D levels $(>30 \mathrm{ng} / \mathrm{mL})$ which included $96 \%$ male and $4 \%$ female. The mean $25(\mathrm{OH})$ vitamin D level in the male severe vitamin D deficiency group was $9.79 \pm 1.57 \mathrm{ng} / \mathrm{mL}$ which was significantly higher as compared to the female group with $7.48 \pm 1.77 \mathrm{ng} / \mathrm{mL}$ as mean value $(p<0.0001)$.In the moderate Vitamin D deficiency group, there was no statistically significant difference between male and female vitamin D level.

\begin{tabular}{|c|c|c|c|c|}
\hline Participants & Moderate deficiency & Severe deficiency & Total & Chi square \\
\cline { 1 - 4 } Male & $26(53 \%)$ & $40(60.60 \%)$ & 66 & \multirow{2}{*}{654} \\
\cline { 1 - 3 } Female & $23(47 \%)$ & $26(39.40)$ & 49 & Not significant \\
\hline Total & 49 & 66 & 115 & \\
\hline
\end{tabular}

This result is not significant at $\mathrm{p}<.05$.

Average 25(OH)D levels did not differ by gender among overweight, severe and moderate obesity or nonoverweight group participants.Figure:1

\section{Gender Distribution}

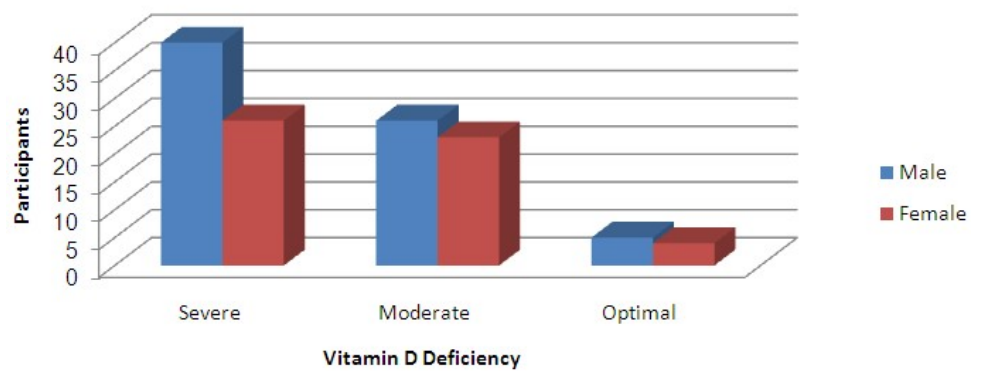


The overall prevalence of obesity was $66.66 \%$. Among Subjects in optimal Vitamin D level it shows a prevalence of $22.22 \%$ in Severe Obesity group, hence severe obesity could be considered as an associated factor for vitamin $\mathrm{D}$ deficiency. Our research result supports the research finding of Rock $\mathrm{Cl}$ et al 2012, involved data from 383 overweight or obese women who participated in a 2-year clinical trial of a weight-loss program which showed that those who did not lose weight at 24 months had an increase in serum 25D of $1.9 \mathrm{ng} / \mathrm{mL}(4.8 \mathrm{nmol} / \mathrm{L})$. However, 25D increased by $2.7 \mathrm{ng} / \mathrm{mL}(6.8 \mathrm{nmol} / \mathrm{L})$ for those who lost $5 \%-10 \%$ of baseline weight, and by $5.0 \mathrm{ng} / \mathrm{mL}(12.5 \mathrm{nmol} / \mathrm{L})$ for those who lost $>10 \%$ of baseline weight $(\mathrm{P}=0.014)$ Early, smaller studies (Liely et al 1988) also reported an association between obesity and low serum 25D concentrations, as well as high concentrations of parathyroid hormone (PTH) and 1,25-dihydroxyvitamin D (1,25D).

The overall participants CRP level ranged from .2 to $15.93 \mathrm{mg} / \mathrm{L}$ with the Mean $5.15 \pm 2.98$, where only $7.32 \%$ of the participants optimal level of CRP, average risk participants were $24.39 \%$ (HSCRP level between $1.0 \mathrm{mg}$ ? L and $3 \mathrm{mg} / \mathrm{L}$ ), and the highest percentage $68.29 \%$ of subjects $(>3 \mathrm{mg} / \mathrm{L})$ were in high risk group. The participants of obesity group had their high level of HSCRP with the Mean 7.07 \pm SD .83, however there is no statistical significant differences among gender distribution of low level vitamin D, HSCRP linked to severe obesity.

Two-way ANOVA was used to compare 25(OH) D levels in the obese group to the non-overweight group, compare seasonal levels, and assess the interaction between obesity and season. Gender patterns were also assessed with two-way ANOVA models.

The interquartile ranges of $25(\mathrm{OH}) \mathrm{D}$ levels for specified subgroups(severe vitamin $\mathrm{D}$ deficiency) is $X_{1}$ 5.75, $X_{u}$ 8.51, $X_{u^{-}} X_{l}=$ 2.725.Box Whisker plot Calculation: Minimum 4.2Maximum 10.23

First quartile 4.5Median 8.04Third quartile 13.5Ascending order 4.200 .

Subjects were classified as low (L) if the serum level of CRP was $<1 \mathrm{mg} / \mathrm{dL}$ or as high $(\mathrm{H})$ if the serum level of CRP was $\geq 1 \mathrm{mg} / \mathrm{dL}$. The detection sensitivity of the assay was $0.1 \mathrm{mg} / \mathrm{dL}$
Among participants $9.75 \%$ were only having normal weight. Among 124 adults, 40.81\% were overweight and $34 \%$ were Obese. A prevalence of $15.44 \%$ among the participants was found to be in Severe Obesity. Study revealed with the great statistics of only $5.55 \%$ were observed to be in low risk HSCRP, $11.11 \%$ of average risk participants, and the higher proportion of the subjects was in high risk group of $83.33 \%$. Average and High risk subjects were with the mean value of 7.44(SD2.59).

In summary increased BMI was positively associated with unfavorable biomarkers, such as LDL, HDL and TG. Similar association between obesity and biochemical markers has been showed in multiple populations worldwide. In obese adult, Pearson correlation co-efficient was used to evaluate the relationship between $25(\mathrm{OH}) \mathrm{D}$ and 2-h glucose, HgbA1c, HSCRP. Data were adjusted for BMI Z score and age. When data did not meet tests of normality, data were log-transformed or nonparametric tests were used. A of 0.05 was considered significant for all statistical tests.

Discussion: Our study described the prevalence of vitamin $\mathrm{D}$ deficiency and the associated link between Obesity and HSCRP among Indian Adults at different referral clinic. In our study the percent change in hsCRP correlated similarly with changes in all measures of body fat, and total abdominal, subcutaneous abdominal, and visceral fat. We have found high prevalence of vitamin D severe deficiency $53.69 \%$, overweight $40.88 \%$, obesity $66.66 \%$, and HSCRP. In addition obese adults are more likely to present HSCRP with vitamin D deficiency and undesirable biochemical markers values than NonObese. Only $9.5 \%$ of the study subjects found to be having normal weight. Vitamin D status may be a determining factor of systemic inflammation in patients with T2DM. ${ }^{[9]}$. In support of this notion we found that the highest proportion of the subjects was observed in high risk group of $83.33 \%$ in comparison with their level of HSCRP, which is alarming sign of leading them to potential cardiovascular risk factors.

The link between obesity and vitamin D deficiency appears to be a one-way street. A large study of the genetics underpinning both conditions finds that obesity may drive down vitamin $\mathrm{D}$ levels, but a predisposition to the vitamin deficiency doesn't lead to obesity. The findings also suggest that boosting vitamin D levels won't reverse obesity. The data suggest that losing weight could potentially reverse 
vitamin D deficiency in obese people, says Hyppönen. Or, she says, obese people could improve their overall health by having their vitamin $\mathrm{D}$ checked and correcting any deficiency. Vitamin D is important for, among other things, fighting infection, absorbing calcium and maintaining a healthy immune system. In summary overweight was positively associated with low level vitamin D and High level SCRP. Low level vitamin D is not significantly associated with Overweight and HSCRP. Further to this our study found the positive associated significant relationship between Obesity and undesirable biomarker values.

Recommendation: Lifestyle intervention would bring the adults the knowledge about the sources of vitamin $\mathrm{D}$ for their better health to prevent them from suffering with vitamin d deficiency related diseases and complications in their lifetime. Prevention (VD supplements) is better than cure and management of sources of acquiring and synthesizing VD is the key, preventing Saudi adolescents from widespread bone and other vitamin D deficiency disorder.

Conclusions: Vitamin D deficiency is common in adults in this southern Indian region and is significantly more prevalent in obese adults. Lower $25(\mathrm{OH}) \mathrm{D}$ level is associated with risk factors for type 2 diabetes in obese adults. This rise in obesity prevalence has paralleled increases in adult hypertension, hyperlipidemia, and type 2 diabetes .Furthermore, epidemiological data have linked low vitamin $\mathrm{D}$ levels to type 2 diabetes The mechanisms by which obesity and its co morbidities are related to vitamin D deficiency are poorly understood. In the present study, we determined the prevalence of vitamin $\mathrm{D}$ deficiency in adults referred to an obesity and compared this prevalence to that of an age, Obesity group and Non obese adults. We have also examined associations between 25-hydroxyvitamin D $[25(\mathrm{OH})$ vitamin $\mathrm{D}]$ and dietary habits in obese adults and tested whether there were correlations between $25(\mathrm{OH})$ vitamin $\mathrm{D}$ and markers of lipid, renal and insulin resistance.

\section{Conflict of Interest:}

The author declares no conflict of interest.

\section{References:}

1. Vimeswaran K., Berry D., Lu C., Pilz S., Hiraki L., Cooper J., Dastani Z., Li R., Houston D., Wood A. Causal relationship between obesity and vitamin D status: Bi-directional mendelian randomization analysis of multiple cohorts. 2013. PLoS Med. 10:1549-1676

2. Walsh J S1, Bowles S, Evans A L, Vitamin D in obesity. Current Opinion in Endocrinology Diabetes and Obesity. 2017. US National Library of Medicine, National Institutes of Health, Volume 24(6), 389-394.

3. Kaptoge, S.; Angelantonio, E.; Pennells, L.; Wood, A. M.; White, I. R.; Gao, P.; Walker, M.; Thompson, A.; Sarwar, N.; Caslake, M.; et al. Creactive protein, fibrinogen, and cardiovascular disease prediction. 2012. The New journal of England Medicine. 4:367, P-1310-1320.

4. Chen N, Wan Z, Han S F, Li B Y, Zhang Z L, Qin L Q, Effect of vitamin D supplementation on the level of circulating high-sensitivity C-reactive protein: a meta-analysis of randomized controlled trials. Nutrients, 2014.US National Library of Medicine, National Institute of Health. 10:6(6).

5. Simon Van lit, Obesity and Vitamin D, 2013. US National Library of Medicine, MDPI, Nutrients, Volume 5; (3) P; 949-956.

6. Vitamin 2 D council 2015. https://www.vitamindcouncil.org/2015-vitamin-dcouncil-annual-report/.

7. Hussain A N, Alkhenizan A H, El Shaker M, Raef $\mathrm{H}$, Gabr A, (2014). Increasing trends and significance of hypovitaminosis D: A populationbased study in the kingdom of Saudi Arabia; (9)190; e PubMed.

8. Pludowski P, karczmarewicz E, Bayer M, Carter G, Chlebna-Sokol D, Czech-Kowalska J, Debski R, Decsi T, Dobrzanska A, Franek E, Gluszko P, Grant WB, Holick MF, Yankovskaya L et al, 2013, US National Library of Medicine, National Institutes of Health, Endokrynologia polska, 64: (4), 319-27.

9. Fatemeh Haidari, Mehrnoosh Zakerkish, Majid Karandish, Azadeh Saki, Sakineh Pooraziz, 2016, Association between serum vitamin D level and Glycemic and Inflammatory markers in Non Obese patients with Type 2 diabetes. Iran Journal of Medical science, 41(5).

10. Erin S. LeBlanc, Joanne H. Rizzo, Kathryn L Pedula, Kristine E. Ensrud, Jane Cauley, Marc Hochberg and Teresa A Hiller, 2012, Association between 25- Hydroxyvitamin $\mathrm{D}$ and weight gain in Elderly women, Journal of women's health, 21(10), 1066-1073. 